\title{
AVALIAÇÃO DA SATISFAÇÃO DOS USUÁRIOS DAS FARMÁCIAS DO SISTEMA PÚBLICO DE SAÚDE
}

\section{User satisfaction assessment in pharmacies of the public health system \\ Evaluación de la satisfacción de los usuarios de las farmacias del sistema público de salud}

\section{Vanessa Adelina Casali Bandeira}

Universidade Regional do Noroeste do Estado do Rio Grande do Sul - UNIJUÍ - Ijuí (RS) -Brasil.

\author{
Aline Schneider \\ Secretaria Municipal de Saúde - SMS - Ijuí (RS) - Brasil.
}

\section{Janaína Barden Schallemberger}

Universidade Regional do Noroeste do Estado do Rio Grande do Sul - UNIJUÍ - Ijuí (RS) - Brasil.

\section{Maristela Codinotti}

Secretaria Municipal de Saúde - SMS - Ijuí (RS) - Brasil.

\section{Marilei Uecker Pletsch}

Universidade Regional do Noroeste do Estado do Rio Grande do Sul - UNIJUÍ - Ijuí (RS) - Brasil.

\section{Christiane de Fátima Colet}

Universidade Regional do Noroeste do Estado do Rio Grande do Sul - UNIJUÍ - Ijuí (RS) - Brasil.

\section{RESUMO}

Objetivo: Avaliar o acesso aos medicamentos e o grau de satisfação dos usuários de farmácias públicas do município de Ijuí, Rio Grande do Sul. Métodos: Trata-se de um estudo observacional, transversal, analítico, de abordagem quantitativa, realizado com usuários das farmácias de 14 unidades de saúde do município em estudo, selecionados aleatoriamente na sala de espera das unidades durante os meses de janeiro e fevereiro de 2013. A coleta de dados aconteceu na unidade de saúde através de questionário semiestruturado, com perguntas referentes a aspectos socioeconômicos, condições de saúde, uso do serviço de saúde e medicamentos. Mensurou-se a satisfação com nota de zero a dez, e os dados foram analisados pelos testes de Mann-Whitney, ANOVA e Kruskal-Wallis. Resultados: Participaram 134 usuários, sendo $75,4 \%(n=101)$ do sexo feminino e idade média de 48,95 16,28 anos. A maioria $(n=89-66,4 \%)$ dos participantes relatou fazer uso contínuo de medicamentos, $54,5 \%(\mathrm{n}=73)$ informou acessar todos os medicamentos na farmácia da respectiva unidade e, na falta destes, $74,6 \%(\mathrm{n}=100)$ informaram comprá-lo. O principal problema apontado nas farmácias foi a falta de medicamentos $(\mathrm{n}=50-37,3 \%)$, seguida pelo atendimento e a falta de recursos humanos (n=17 - 12,6\%). A satisfação média com os serviços ofertados pela farmácia foi de $8,7 \pm 1,5$. Verificou-se maior satisfação entre os participantes de menor escolaridade, que têm acesso aos medicamentos e as dúvidas esclarecidas. Conclusão: Os resultados indicam satisfação com os serviços prestados, porém os achados reportam insuficiência no acesso aos medicamentos e necessidade de efetiva qualificação da assistência farmacêutica para a garantia do acesso ao tratamento, com qualidade e resolutividade.

Descritores: Assistência Farmacêutica; Acesso aos Serviços de Saúde; Satisfação do Paciente; Uso de medicamentos.

\section{ABSTRACT}

Objective: To assess the access to medicines and the level of satisfaction in users of public pharmacies in the municipality of Ijui, Rio Grande do Sul. Methods: Quantitative observational and analytical cross-sectional study conducted with users of pharmacies from 14 health care centers who were randomly selected in the waiting room in January and February 2013. Data were collected using a semi-structured questionnaire on socioeconomic aspects, health conditions, and use of health services and medicines. Satisfaction was measured by a score of zero to 10. Data were analyzed using the Mann-Whitney, ANOVA and Kruskal-Wallis tests. Results: Participants were 134 users: 75.4\% $(n=101)$ of them were women and mean age was $48.95 \pm 16.28$ years. Most $(n=89-66.4 \%)$ of the participants reported long term use of medicines, $54.5 \%(n=73)$ reported access to all the medicines in the pharmacy of the center, and $74.6 \%$ (100) reported buying the medicine if the pharmacy was out of them. The main problem identified was the lack of medicines ( $n=50-37.3 \%)$, followed by service and lack of human 
resources ( $n=17-12.6 \%$ ). Mean satisfaction with the services was $8.7 \pm 1.5$. Greater satisfaction was found in those with lower levels of education, those who have access to medicines and those who have their doubts clarified. Conclusion: There is satisfaction with the services provided; however, the findings highlight the poor access to medicines and the need for effective qualification of the pharmaceutical services provided to ensure access to treatment with quality and problem-solving capacity.

Descriptors: Pharmaceutical Services; Health Services Accessibility; Patient Satisfaction; Drug Utilization.

\section{RESUMEN}

Objetivo: Evaluar la accesibilidad a los medicamentos y el grado de satisfacción de los usuarios de las farmacias públicas del municipio de Ijui/Rio Grande do Sul. Métodos: Se trata de un estudio observacional, transversal, analítico y de abordaje cuantitativo realizado con los usuarios de las farmacias de 14 unidades de salud del municipio del estudio elegidos de manera aleatoria en la sala de espera de las unidades durante los meses de enero y febrero de 2013. La recogida de datos se dio en la unidad de salud a través de un cuestionario semi estructurado con preguntas sobre los aspectos socioeconómicos, las condiciones de salud, el uso del servicio de salud e los medicamentos. La satisfacción fue medida de cero a diez. Los datos fueron analizados por las pruebas de Mann-Whitney, la ANOVA y la de Kruskal-Wallis. Resultados:

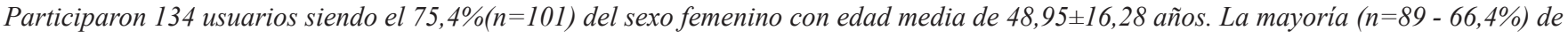
los participantes ha relatado el uso continuo de medicamentos, el 54,5\% ( $n=73)$ informó que tuvo la accesibilidad a los medicamentos en la farmacia de la respectiva unidad y que, en su ausencia, el 74,6\% (n=100) los compraba. El principal problema de las farmacias fue la falta de medicamentos $(n=50-37,3 \%)$, seguida de la atención y la falta de recursos humanos $(n=17-12,6 \%)$. La satisfacción media con los servicios ofrecidos por la farmacia fue de $8,7 \pm 1,5$. Se verificó más satisfacción entre los participantes con menos escolaridad que tienen el acceso a los medicamentos y que tuvieron sus dudas aclaradas. Conclusión: Los resultados indican la satisfacción con los servicios ofrecidos, sin embargo, los hallazgos señalan la insuficiencia para el acceso a los medicamentos y la necesidad de una efectiva cualificación de la atención farmacéutica para garantizar el acceso al tratamiento de calidad y la solución del problema.

Descriptores: Servicios Farmacéuticos; Accesibilidad a los Servicios de Salud; Satisfacción del Paciente; Utilización de Medicamentos.

\section{INTRODUÇÃO}

A assistência farmacêutica (AF), como política pública, foi organizada e fortalecida por meio da Política Nacional de Medicamentos (PNM), publicada em 2001, visando garantir a segurança, a eficácia e a qualidade dos medicamentos; a promoção do uso racional e o acesso àqueles considerados essenciais ${ }^{(1)}$. Na configuração atual do Sistema Único de Saúde (SUS), a AF é um componente determinante para a resolubilidade da atenção e contribui de forma decisiva no controle de doenças e aumento da expectativa e qualidade de vida da população. Para que seja efetiva, no entanto, são necessários recursos financeiros, físicos, humanos e tecnológicos, adequados e compatíveis, em cada território ${ }^{(2)}$.

Nesse contexto, o acesso aos medicamentos nos serviços públicos de saúde do Brasil foi ampliado nos últimos anos, subindo de 26,40\% (em 2003) para 48,55\% (em 2008), como demonstrou inquérito domiciliar realizado no município de São Paulo. O estudo também verificou maior contingente de acesso pela população com menor poder aquisitivo e a expansão do acesso público aos medicamentos pela população com maior renda. Destaca-se que a cobertura universal para gastos com medicamentos essenciais e a equidade no acesso aos medicamentos ainda são desafios para o SUS, uma vez que a falta de acesso e o uso irracional de medicamento colocam em risco os investimentos das ações de saúde ${ }^{(3)}$.

Outro fator importante é a indisponibilidade de medicamentos nos serviços de saúde, que pode estar correlacionada à: menor adesão ao tratamento medicamentoso ${ }^{(4-7)}$, pior estado de saúde, aumento dos gastos com tratamento, maior uso dos serviços de saúde e também de terapias adicionais, interferindo na satisfação dos usuários desses serviços ${ }^{(4,8)}$. A execução inadequada das últimas etapas da AF, tais como na dispensação e orientação farmacêutica, também repercutem para o uso irracional dos medicamentos e insatisfação com a AF prestada. Um estudo realizado nas farmácias públicas de Ribeirão Preto, São Paulo ${ }^{(5)}$ identificou alta prevalência de idosos com posologia incorreta de seus medicamentos, relacionando com a falta de informação sobre o uso dos mesmos. Além disso, nas unidades de saúde de Minas Gerais, constatou-se que esse desconhecimento influencia diretamente a não adesão do paciente hipertenso à farmacoterapia ${ }^{(8)}$.

$\mathrm{O}$ uso de medicamento é uma ferramenta importante para o cuidado à saúde da população, por auxiliar na promoção, proteção e recuperação da saúde e prevenção de agravos ${ }^{(1)}$. É indiscutível, nesse contexto, a importância de conhecer os serviços públicos que ofertam medicamentos e a satisfação de seus usuários, a fim de se obter informações que norteiem o planejamento de ações das equipes de saúde e qualifiquem o cuidado $^{(9)}$.

Diante dessas considerações, busca-se, no presente estudo, avaliar o acesso aos medicamentos e o grau de satisfação dos usuários de farmácias públicas do município de Ijuí, Rio Grande do Sul. 


\section{MÉTODOS}

Trata-se de um estudo observacional, transversal e analítico, de abordagem quantitativa, realizado com usuários das farmácias públicas do município de Ijuí, Rio Grande do Sul. A AF municipal é constituída por: uma central de medicamentos, uma farmácia de atendimento exclusivo para medicamentos de controle especial, uma farmácia de medicamentos especializados, uma farmácia do Serviço de Atendimento Especializado (SAE), uma farmácia de medicamentos estratégicos e 14 farmácias das unidades de saúde de medicamentos básicos.

A amostra do estudo foi composta intencionalmente, com seleção de 10 usuários de cada farmácia pública, totalizando 134 usuários dos serviços supracitados, escolhidos aleatoriamente na sala de espera dos referidos locais durante os meses de janeiro e fevereiro de 2013. Incluíram-se os usuários com idade igual ou superior a 18 anos e de ambos os sexos, sendo excluídos os pacientes que não conseguiram responder aos questionamentos feitos pelas pesquisadoras.

A coleta de dados ocorreu por meio de um questionário semiestruturado, constituído de perguntas abertas e fechadas referentes aos aspectos sociodemográficos (como sexo, idade e escolaridade), condições de saúde (como presença de doenças crônicas), uso e acesso aos serviços de saúde e medicamentos. Para a realização da pesquisa, os entrevistadores, todos acadêmicos do curso de farmácia, foram previamente treinados e o questionário foi validado por um estudo-piloto.

Avaliou-se a satisfação dos usuários com o serviço da farmácia através de escala de zero a dez, sendo a última a nota máxima e indicativa de melhor satisfação. $\mathrm{O}$ uso de medicamentos foi mensurado conforme o autorrelato dos entrevistados. Considerou-se uso contínuo de medicamentos aqueles que referiram usar de forma ininterrupta, sem informação de tempo para o fim do tratamento. Os dados obtidos foram compilados em tabelas por meio do software Statistical Package for the Social Sciences (SPSS), versão 18.0, em que empregaram-se recursos de análise descritiva simples, tais como média, desvio padrão, valor mínimo e máximo. Para verificar a normalidade das variáveis, aplicou-se o teste de Kolmogorov-Smirnov; utilizou-se o teste de Mann-Whitney para comparação de médias para amostras não paramétricas e independentes; e, para comparações de médias dos escores dos vários grupos, empregou-se o teste de ANOVA e Kruskal-Wallis. Para todos os testes considerou-se estatisticamente significativo $\mathrm{p} \leq 0,05$.

Na construção do presente trabalho, observaram-se todos os preceitos éticos preconizados para pesquisa com seres humanos e recebeu aprovação pelo Comitê de Ética em Pesquisa sob parecer consubstanciado nº 176.210/2012.

\section{RESULTADOS}

A amostra foi composta por 134 usuários, sendo 75,4\% (n=101) do sexo feminino, com idade média de 48,95 16,28 anos (em que a mínima de 19 anos e máxima de 95 anos), faixa etária predominante acima de 60 anos (29,1\% - n=39), e com oito anos ou mais de escolarização $(50,7 \%$ - $n=68)$ conforme apresentado na Tabela I.

Tabela I - Dados sociodemográficos dos usuários de farmácias das unidades de saúde de Ijuí, Rio Grande do Sul, 2013 (n=134).

\begin{tabular}{lcc}
\hline Variáveis & n & \% \\
\hline Sexo & 101 & 75,4 \\
$\quad$ Feminino & 33 & 24,6 \\
$\quad$ Masculino & & \\
Faixa etária & 16 & 11,9 \\
$19-29$ anos & 29 & 21,7 \\
$30-39$ anos & 24 & 17,9 \\
$40-4$ anos & 26 & 19,4 \\
$50-59$ anos & 39 & 29,1 \\
$\geq 60$ anos & & 49,3 \\
Escolaridade & 66 & 50,7 \\
$\quad<8$ anos de escolarização & 68 & \\
$\geq 8$ anos de escolarização & & \\
\hline
\end{tabular}

Quanto ao uso de medicamentos, a maioria $(66,4 \%$ - $n=89)$ informou fazer uso contínuo deles. Dentre eles, $89,9 \%(n=80)$ referiram ser acometidos por alguma doença crônica.

Em relação ao acesso aos medicamentos prescritos, 54,5\% $(\mathrm{n}=73)$ informaram conseguir todos os medicamentos na farmácia da unidade de saúde, $29,1 \%(\mathrm{n}=39)$ não conseguem e 16,4\% $(\mathrm{n}=22)$ conseguem às vezes. Quando analisada a relação entre $\mathrm{o}$ acesso e as características sociodemográficas, sexo $(\mathrm{p}=0,879)$, faixa etária $(\mathrm{p}=0,094)$ e escolaridade $(\mathrm{p}=0,118)$, não se identificaram diferenças significativas.

Quando questionados sobre o conhecimento da indicação dos medicamentos prescritos, 85,1\% ( $\mathrm{n}=114)$ afirmaram saber a indicação e $87,3 \%$ (n=117) relataram que suas dúvidas são esclarecidas na farmácia da unidade. Na falta de medicamentos, 
$74,6 \%(n=100)$ informaram comprar o medicamento, 14,2\% $(n=19)$ citaram o programa Farmácia Popular, 8,9\% $(n=12)$ retiram em outra unidade, $3,7 \%(n=5)$ esperam vir o medicamento e $0,7 \%(n=1)$ informou que acessa via judicial. A falta de medicamento foi apontada como o principal problema nas farmácias por 37,3\% $(n=50)$ dos entrevistados e 50,7\% $(n=68)$ relataram que sua ocorrência é frequente, como evidenciado na Tabela II.

Tabela II - Avaliação dos usuários sobre os problemas e as mudanças necessárias nas farmácias das unidades de saúde de Ijuí, Rio Grande do Sul, 2013, (n=134).

\begin{tabular}{lcc}
\hline Variáveis & $\mathbf{n}$ & $\mathbf{\%}$ \\
\hline Problemas na farmácia da unidade básica de saúde & & 45,5 \\
Não tem problemas & 61 & 37,3 \\
Falta de medicamentos & 50 & 12,6 \\
Atendimento/Recursos humanos & 17 & 4,6 \\
Estrutura Física & 6 & 50,7 \\
A falta de medicamentos é comum? & 68 & 29,1 \\
Sim & 39 & 21,6 \\
Não & 29 & 49,3 \\
Às vezes & & 31,3 \\
O que você gostaria que fosse diferente? & 66 & 15,7 \\
Nada, está bom assim/ Não sabe & 42 & 3,7 \\
Que tivesse todos os medicamentos & 21 & 5 \\
Atendimento/Recursos humanos & & \\
Melhor estrutura física & 5 &
\end{tabular}

A satisfação com o serviço das farmácias estudadas apresentou avaliação média de $8,7 \pm 1$,5, com mínima de 3 e máxima de 10. A avaliação das farmácias públicas apresentou-se relacionada à escolaridade, ao acesso ao medicamento e ao esclarecimento de dúvida dos usuários. Constatou-se maior satisfação entre os participantes com menor escolaridade, com média de 9,1 $\pm 1,58$, enquanto a média daqueles com maior escolaridade foi de $8,4 \pm 1,46(\mathrm{p}=0,000)$. Quanto ao acesso aos medicamentos, a maior média foi identificada entre os que referiram acesso total aos medicamentos nas farmácias públicas, seguidos daqueles com acesso parcial e sem acesso, respectivamente, $9,0 \pm 1,38,8,45 \pm 1,54$ e $8,36 \pm 1,87(p=0,039)$, com diferença significativa entre os participantes com acesso total e parcial $(\mathrm{p}=0,033)$. Em relação ao esclarecimento das dúvidas, os resultados demostraram maior satisfação daqueles que têm suas dúvidas esclarecidas, com média de $8,9 \pm 1,41$, e de 7,59 2,0 para aqueles que não têm as dúvidas esclarecidas $(\mathrm{p}=0,007)$.

\section{DISCUSSÃO}

A satisfação com os serviços ofertados pelas farmácias públicas avaliadas no atual estudo apresentou média elevada, o que pode indicar, de acordo com a avaliação dos participantes, que o atendimento prestado está adequado. No entanto, a falta de medicamentos foi apontada como o principal problema das farmácias estudadas. Constatou-se a relação entre o acesso aos medicamentos e a satisfação com os serviços, com maior satisfação dos participantes que têm acesso a todos os medicamentos. Destaca-se também que, como a coleta de dados foi realizada na unidade de saúde, os participantes podem ter omitido informações por insegurança ou medo de não serem atendidos. Além disso, os participantes que informaram que suas dúvidas são esclarecidas nas farmácias das unidades de saúde também apresentaram notas mais elevadas em satisfação.

Entre as variáveis sociodemográficas, os participantes com maior escolaridade referiram menores notas de satisfação com o serviço. Um estudo desenvolvido na Região Metropolitana de São Paulo apontou que a educação é uma variável correlacionada ao uso dos serviços de saúde e seu efeito é maior do que a renda individual, uma vez que os indivíduos com maior nível educacional utilizam mais os serviços de saúde por cuidados preventivos ${ }^{(10)}$. Esses achados demonstram maior conhecimento sobre questões de saúde, o que consequentemente pode influenciar na avaliação de seus serviços.

As demais características sociodemográficas não se apresentaram como características associadas ao acesso aos medicamentos e à satisfação com os serviços prestados. Entre essas características, verificou-se o predomínio de mulheres, semelhante a outros estudos realizados nos serviços de saúde brasileiros ${ }^{(7,9,12)}$, assim como a baixa escolaridade, inferior a oito anos de estudo ${ }^{(7,12,13)}$. Quanto à média de idade, foi superior aos estudos realizados em Belo Horizonte, Minas Gerais ${ }^{(14)} \mathrm{e}$ Blumenau, Santa Catarina ${ }^{(7)}$, os quais identificaram 39,2 e 43,3 anos respectivamente. Esses resultados demonstram que, apesar do maior consumo de medicamentos por idosos, indivíduos de menor faixa etária também fazem uso contínuo de medicamentos e reforçam a necessidade de orientações, tanto para o adequado tratamento medicamentoso quanto não medicamentoso, das doenças relacionadas. 
Condições demográficas, sociais e econômicas, tais como: sexo feminino, faixa etária mais avançada, presença de doenças crônicas, baixa escolaridade e maior renda, estão relacionadas ao maior uso dos serviços de saúde e de medicamentos ${ }^{(9,13)}$, influenciando no acesso aos medicamentos e na adesão à terapia medicamentosa, assim como evidenciado em outros estudos ${ }^{(6,7)}$. Identificou-se entre idosos de Bagé, Rio Grande do Sul, a baixa adesão ao tratamento associada à idade mais avançada, ocorrência de três ou mais morbidades, e a necessidade de compra total ou parcial de medicamento ${ }^{(4)}$. Os autores apontam a importância do acesso aos medicamentos, uma vez que a maioria da população atendida no serviço público de saúde é de baixa renda e a obtenção gratuita frequentemente é a única alternativa de acesso. Nesse sentido, destaca-se que o medicamento é parte importante da atenção à saúde e uma das ferramentas terapêuticas mais utilizadas para tratar doenças, promover qualidade de vida e prevenir complicações e epidemias ${ }^{(15)}$. No Brasil, o SUS, através das políticas de AF, busca promover o acesso da população aos medicamentos considerados essenciais, ou seja, que atendem a maioria dos agravos e doenças e às ações prioritárias sobre a saúde da população(2).

A maioria dos entrevistados referiu o uso contínuo de medicamentos na atual pesquisa, no entanto, quase a metade dos participantes não tem acesso total ao seu tratamento, o que demonstra dificuldade de acesso, semelhante ao identificado na Pesquisa Nacional por Amostra de Domicílios (PNAD), com 19.427 entrevistados de cinco regiões do país, dos quais 45,3\% receberam todos os medicamentos e $18,7 \%$ parcialmente ${ }^{(12)}$. Estudos com adultos das Regiões Sul e Nordeste ${ }^{(16)}$ do país identificaram acesso total por 36,6\% e parcial para 5,1\% dos participantes. Verificou-se, em uma unidade de saúde de Porto Alegre, Rio Grande do Sul ${ }^{(17)}$, que $86,75 \%$ das prescrições foram totalmente atendidas e $13,25 \%$ parcialmente. Em Ribeirão Preto, São Paulo, constatou-se que $46,8 \%$ conseguiram todos os seus medicamentos pelo SUS ${ }^{(5)}$.

Entre as causas relacionadas à falta de acesso aos medicamentos, um estudo com usuários da atenção primária de Blumenau apontou que $25,0 \%$ dos indivíduos não obtiveram todos os medicamentos prescritos e, entre os principais motivos da não obtenção, encontrava-se "o medicamento não é fornecido pelo SUS" $(42,5 \%)$ e "ausência do medicamento no estoque da farmácia" $(40,7 \%)^{(7)}$. Além disso, uma pesquisa em Ribeirão Preto identificou que 31,3\% dos idosos usuários das farmácias do SUS não conseguiram obter todos os seus medicamentos prescritos, os quais estavam em falta no momento da retirada na farmácia ${ }^{(5)}$, apesar de estarem padronizados na Relação Municipal de Medicamentos Essenciais (REMUME).

A REMUME é um instrumento norteador das prescrições. Quando os medicamentos que a compõem são corretamente selecionados e sua adoção ocorre de forma efetiva pelos prescritores, auxilia na programação e facilita o acesso aos medicamentos prescritos ${ }^{(18)}$. Considera-se, no entanto, que a REMUME é composta por medicamentos considerados essenciais, que atendem a maioria dos problemas que acometem a população e, conforme recomendado pela Organização Mundial de Saúde ${ }^{(18)}$, no mínimo $70 \%$ dos medicamentos prescritos devem estar presentes na REMUME, condição que pode gerar algumas lacunas para o tratamento de determinadas doenças. Além disso, a falta de medicamentos presentes na REMUME nos serviços municipais pode ser decorrente de recursos financeiros insuficientes, planejamento inadequado ou problemas na distribuição as farmácias públicas. No presente estudo, não foi avaliado se os medicamentos não encontrados nas farmácias públicas pesquisadas pertenciam à REMUME, o que se apresenta como uma limitação do estudo.

Destaca-se que o acesso insuficiente aos medicamentos está diretamente associado à piora do estado de saúde, à necessidade de novos tratamentos, ao aumento no número de consultas e aos gastos adicionais ${ }^{(15)}$. Nesse contexto, para acesso ao medicamento diante de sua indisponibilidade nas farmácias públicas, a compra foi apontada pela maioria dos entrevistados na presente investigação, semelhante ao identificado em estudos nacionais ${ }^{(4,12)}$. O programa Farmácia Popular do Brasil também foi referido pelos participantes da atual pesquisa, o qual é uma estratégia do Ministério da Saúde com vistas a ampliar o acesso aos medicamentos para as doenças prevalentes na população, seja de forma gratuita, seja $90 \%$ mais baratos, na rede privada de farmácias e drogarias ${ }^{(19)}$. Identificou-se entre os entrevistados da atual pesquisa que o programa Farmácia Popular é uma ferramenta de acesso aos medicamentos quando não estão disponíveis nas farmácias das unidades, o que auxilia na manutenção da terapia medicamentosa e na adesão ao tratamento.

Além disso, cinco participantes da pesquisa em questão relataram esperar vir o medicamento, o que representa descontinuidade e não adesão ao tratamento, bem como possíveis riscos para agravos de sintomas e complicações da doença. Uma pesquisa observou que $8,8 \%$ dos entrevistados das cinco regiões do país não adquiriram os medicamentos e destaca que a não aquisição relaciona-se com a classe social (classes socioeconômicas mais baixas), grau de instrução (menor escolaridade), raça (negros) e faixa etária (jovens e adultos) ${ }^{(12)}$. Cabe ressaltar que os participantes da pesquisa foram questionados sobre como adquiriam os medicamentos na falta deles na farmácia pública, o que pode influenciar para uma resposta mais resolutiva.

A falta de medicamentos foi referida como frequente e apontada como o principal problema das farmácias públicas pesquisadas no presente estudo, seguida por recursos humanos e estrutura física. Em estudo realizado em cinco municípios do estado de São Paulo, verificaram como principal queixa a falta de medicamentos ${ }^{(20)}$. De maneira semelhante, em um município de Sergipe, entre as reclamações do serviço público, constaram: falta de medicamentos, infraestrutura precária, burocracia, relação médico-paciente insatisfatória, falta de cortesia e atenção por parte de alguns profissionais da saúde, entre outras ${ }^{(21)}$.

Nesse contexto, como consequência da insuficiência de acesso aos medicamentos, ocorre redução da adesão ao tratamento medicamentoso $^{(6)}$. Destaca-se que o acesso consiste na primeira etapa de uma AF de qualidade e que, para que seja efetiva, são necessárias melhorias no que se refere aos cuidados farmacêuticos, para se obter resultados efetivos, com maior adesão e menor quantidade de estoque domiciliar ${ }^{(7)}$. Receber os medicamentos não implica necessariamente em melhores condições de 
saúde ou qualidade de vida, pois os maus hábitos prescritivos e as falhas na dispensação podem levar a tratamentos ineficazes e inseguros ${ }^{(6,7)}$. Essas considerações vêm ao encontro das queixas relacionadas ao atendimento e falta de recursos humanos e informações sobre o medicamento no atual trabalho.

No presente estudo, a maioria referiu receber informações e esclarecer suas dúvidas quanto ao tratamento medicamentoso na farmácia da unidade de saúde, o que difere de estudo realizado em um município baiano, no qual a dispensação restringiase à rápida entrega do medicamento pelo técnico de enfermagem, sem qualquer comunicação ou orientação ao usuário ${ }^{(22)}$. Outros estudos, em uma ESF de Ponta Grossa e entre hipertensos acolhidos em uma ESF no interior de Minas Gerais, também identificaram dados inferiores: $76,2 \%$ e 79,3\%, respectivamente, receberam orientação sobre o uso de medicamentos ${ }^{(8,9)}$. Destaca-se, no entanto, que a coleta de dados nos estudos supracitados foi realizada no domicílio, diferentemente do presente estudo, realizado na unidade de saúde, o que pode ter interferido nas respostas afirmativas quanto ao esclarecimento de dúvidas. Além disso, o recebimento de informações não significa efetivamente seu entendimento, e a resposta no presente estudo foi dada mediante autorrelato, o que constitui outra limitação.

Entre as condições que comprometem a qualidade da AF em vários municípios ${ }^{(23)}$, citam-se os recursos financeiros escassos; falta de instrução de profissionais; processos falhos de seleção de medicamentos; ausência de planejamento e programação para a aquisição de medicamentos; e armazenamento inapropriado de medicamentos. Estudo realizado em vinte municípios da região noroeste do estado do Rio Grande do Sul, revelou problemas nos recursos materiais, financeiros e humanos, principalmente no que se refere à presença do profissional farmacêutico nas farmácias públicas ${ }^{(24)}$. Enquanto que em uma unidade de Porto Alegre Rio Grande do Sul verificou-se o papel do farmacêutico como assessor do medicamento, na equipe de saúde, não condiz com as recomendações vigentes ${ }^{(25)}$.

A dispensação não realizada por profissional farmacêutico dificulta o fornecimento de informações sobre o tratamento, o que pode resultar no uso irracional de medicamentos. A presença do farmacêutico na unidade de saúde melhora a gestão da AF na unidade, principalmente no que se refere à dispensação, orientação e tomada de decisão para condutas terapêuticas. A realização de acompanhamento farmacoterapêutico é uma ferramenta que auxilia na redução das dificuldades de uso dos medicamentos e auxilia para a melhora da adesão e qualidade de vida do usuário(4). Além disso, ao avaliarem a satisfação dos hipertensos com o serviço ofertado na presente pesquisa, $80 \%$ consideraram importante o trabalho conjunto do farmacêutico com o médico e $100 \%$ responderam que continuariam a utilizar o serviço, indicando-o a amigos e parentes.

Destaca-se que o farmacêutico é o profissional capacitado para garantir o acesso da população a medicamentos de qualidade, ofertar serviços farmacêuticos e promover uso racional de medicamentos aos usuários e comunidade, beneficiando-os. A presença do farmacêutico na ESF beneficia os também a equipe de saúde, pois, quando inserido na equipe multiprofissional, pode capacitá-los e auxiliá-los quanto às condutas relacionadas ao uso de medicamentos. Na comunidade, participa de ações de educação e promoção da saúde e garante para os usuários o fornecimento de orientação e informação, auxiliando-os na administração correta de medicamentos e adesão ao tratamento medicamentoso ${ }^{(26)}$.

Avaliar a satisfação dos usuários é, assim, fundamental para melhorar a gestão dos serviços prestados, pois o entendimento da avaliação dos usuários indica decisões estratégicas e operacionais que influenciam o nível de qualidade dos serviços ${ }^{(9)}$. Nesse sentido, em meio aos desafios relacionados às demandas e melhorias da AF no SUS, apresenta-se a necessidade de conscientização de gestores para que se invista em estrutura física, recursos humanos, organização dos processos e capacitação permanente dos trabalhadores envolvidos com as atividades que fazem parte do ciclo da AF, bem como a inserção do profissional farmacêutico nesses locais, que torna o acesso aos medicamentos viável, racional e mais eficiente ${ }^{(23,26)}$.

O presente estudo limita-se por não ter acessado as prescrições dos indivíduos atendidos nas farmácias públicas e avaliado a dispensação dos medicamentos que a compõem, bem como a presença desses medicamentos nas listas oficias do SUS, indicadores importantes para a avaliação da programação, execução e resolutividade da AF.

\section{CONCLUSÃO}

A satisfação dos serviços de saúde apresentou nota superior a oito, o que representa uma avaliação geral positiva dos serviços prestados pelas farmácias das unidades de saúde do município em estudo. Apesar dessa avaliação, identificou-se que apenas metade dos participantes tem acesso a todos os medicamentos prescritos nas farmácias públicas e a falta de medicamentos foi apontada como o problema principal e frequente pelos entrevistados.

Esses resultados reportam a insuficiência do acesso aos medicamentos no SUS, bem como a necessidade de efetivação de todas as etapas da AF, iniciado pela seleção adequada, o apoio de equipe multiprofissional para a prescrição, conforme preconizado, e a dispensação de qualidade, em busca da promoção do uso racional de medicamentos.

\section{REFERÊNCIAS}

1. Secretaria de Políticas de Saúde (BR), Departamento de Atenção Básica. Política Nacional de Medicamento. Brasília: Ministério da Saúde; 2001 [acesso em 2016 Dez 30]. Disponível em: http://bvsms.saude.gov.br/bvs/publicacoes/politica_ medicamentos.pdf 
2. Conselho Nacional de Secretários de Saúde (BR). Atenção Primária e Promoção da Saúde. Brasília: CONASS; 2011. (Coleção Para Entender a Gestão do SUS) [acesso em 2016 Dez 30]. Disponível em: http://www.conass.org.br/colecao2011/ livro_3.pdf

3. Monteiro CN, Gianini RJ, Barros MBA, Cesar CLG, Goldbaum M. Access to medication in the Public Health System and equity: populational health surveys in São Paulo, Brazil. Rev Bras Epidemiol. 2016;19(1):26-37.

4. Tavares NUL, Bertoldi AD, Thumé E, Facchini LA, Fraça GVA, Mengue SS. Fatores associados à baixa adesão ao tratamento medicamentoso em idosos. Rev Saúde Pública. 2013;47(6):1092-101.

5. Baldoni AO, Dewulf NLS, Santos V, Reis TM, Ayres LR, Pereira LRL. Dificuldades de acesso aos serviços farmacêuticos pelos idosos. Rev Ciênc Farm Básica Apl. 2014;35(4):615-21.

6. Remondi FA, Cabrera MAS, Souza RKT. Não adesão ao tratamento medicamentoso contínuo: prevalência e determinantes em adultos de 40 anos e mais. Cad Saúde Pública 2014;30(1):126-36.

7. Helena ETS, Andersen SE, Menoncin SM. Percepção dos usuários sobre acesso aos medicamentos na atenção primária. Cad Saúde Colet (Rio de J). 2015;23(3):280-8.

8. Ferreira FM, Cruz MJB, Santos DF, Linhares MP, Andrade RA. Fatores relacionados à adesão farmacoterapêutica de pacientes hipertensos acolhidos na estratégia de saúde da família. Rev APS. 2013;16(3):258-68.

9. Vosgerau MZS, Soares DA, Souza RKT, Matsuo T, Carvalho GS. Consumo de medicamentos entre adultos na área de abrangência de uma Unidade de Saúde da Família. Ciênc Saúde Coletiva 2011;16(Supl 1):1629-38.

10. Chiavegatto ADP Filho, Wang YP, Malik AM, Takaoka J, Viana MC, Andrade LH. Determinants of the use of health care services: multilevel analysis in the Metropolitan Region of Sao Paulo. Rev Saúde Pública. 2015;49(1):1-12.

11. Reis SR, Coimbra LC, Silva AAM, Santos AM, Alves MTSSB, Lamy ZC, et al. Acesso e utilização dos serviços na Estratégia Saúde da Família na perspectiva dos gestores, profissionais e usuários. Ciênc Saúde Coletiva. 2013;18(11):332131.

12. Boing AC, Bertoldi AD, Boing AF, Bastos JL, Peres KG. Acesso a medicamentos no setor público: análise de usuários do Sistema Único de Saúde no Brasil. Cad Saúde Pública. 2013;29(4):691-701.

13. Bertoldi AD, Dal Pizzol TS, Ramos LR, Mengue SS, Luiza VL, Tavares NUL, et al. Perfil sociodemográfico dos usuários de medicamentos no Brasil: resultados da PNAUM 2014. Rev Saúde Pública. 2016;50(Supl 2):1s-11s.

14. Lima-Costa MF, Loyola AI Filho. Fatores associados ao uso e à satisfação com os serviços de saúde entre usuários do Sistema Único de Saúde na Região Metropolitana de Belo Horizonte, Estado de Minas Gerais, Brasil. Epidemiol Serv Saúde. 2008;17(4):247-57.

15. Wannmacher L. Importância dos medicamentos essenciais em prescrição e gestão racionais. In: Ministério da Saúde (BR), Secretaria de Ciência, Tecnologia e Insumos Estratégicos. Uso racional de medicamentos: temas selecionados. Brasília: Ministério da Saúde; 2012. p. 15-20.

16. Paniz VMV, Flores ICC, Fassa AG, Piccini RX, Tomasi E, Thumé E, et al. Acesso a medicamentos para tratamento de condições agudas prescritos a adultos nas regiões Sul e Nordeste do Brasil. Cad Saúde Pública. 2016;32(4):e00009915.

17. Bueno D, Sampaio GC, Guerin GD. Análise do acesso a medicamentos em uma unidade do programa de saúde da família em Porto Alegre. Rev APS. 2013;16(1):83-9.

18. World Health Organization. How to investigate drug use in health facilities: selected drug use indicators [Internet]. Geneva: WHO; 1993 [acessado em 2016 Dez 30]. Disponível em: URL http://apps.who.int/medicinedocs/pdf/s2289e/s2289e.pdf

19. Brasil. Ministério da Saúde. Gabinete do Ministro. Portaria $\mathrm{n}^{\circ} 1.019$, de 20 de maio de 2014. Altera a Portaria $\mathrm{n}^{\circ} 2.587 / \mathrm{GM} /$ MS, de 6 de dezembro de 2004, que institui o incentivo financeiro do Programa Farmácia Popular do Brasil, e dá outras providências. Brasília; 2014 [acesso em 2016 Dez 30]. Disponível em: http://bvsms.saude.gov.br/bvs/saudelegis/gm/2014/ prt1019_20_05_2014.html.

20. Moimaz SAS, Marques JAM, Saliba O, Garbin CAS, Zina LG, Saliba NA. Satisfação e percepção do usuário do SUS sobre o serviço público de saúde. Physis (Rio de J). 2010; 20(4):1419-40.

21. Souza LS, Porto JG, Lyra DP Júnior. Avaliação da estrutura e dos processos de organização e gestão da assistência farmacêutica em município do estado de Sergipe. Rev Ciênc Farm Básica Apl. 2011;32(3):403-10.

22. Alencar TOS, Alencar BR, Silva DS, Araújo JSC, Oliveira SM, Souza RD. Promoção do uso racional de medicamentos: uma experiência na estratégia saúde da família. Rev Bras Promoç Saúde. 2014;27(4):575-82. 
23. Oliveira LCF, Assis MAA, Barboni AR. Assistência farmacêutica no Sistema Único de saúde: da política nacional de medicamentos à atenção básica á saúde. Ciênc Saúde Coletiva 2010;15(Supl 3):3561-7.

24. Bernardi CLB, Bieberbach EW, Thome HI. Avaliação da assistência farmacêutica básica nos municípios de abrangência da $17^{\text {a }}$ Coordenadoria Regional de Saúde do Rio Grande do Sul. Saúde Soc. 2006;15(1):73-83.

25. Bueno D, Machado AR. Avaliação dos dispensários do distrito sanitário Glória- Cruzeiro-Cristal Porto Alegre-RS. Rev APS 2011;14(1):4-11.

26. Ministério da Saúde (BR), Secretaria de Ciência, Tecnologia e Insumos Estratégicos, Departamento de Assistência Farmacêutica Insumos Estratégicos. Resultados do projeto de implantação do cuidado farmacêutico no Município de Curitiba. Brasília: Ministério da Saúde; 2015. (Cuidado farmacêutico na atenção básica, no 4).

Conflito de interesse: Inexistente.

Suporte financeiro: Não houve.

\section{Endereço para correspondência:}

Vanessa Adelina Casali Bandeira.

Universidade Regional do Noroeste do Estado do Rio Grande do Sul - UNIJUÍ

Departamento de Ciências da Vida

Rua do Comércio, 3000

Bairro: Universitário

CEP 98700-000 - Ijuí - RS - Brasil

E-mail: vanessa.acbandeira@yahoo.com.br 